\title{
Analysis of pregnancy outcome after anastomosis of oviduct and its influencing factors
}

\author{
Yun Feng ${ }^{1,2^{*}+}$, Han Zhao $^{1,2+}$, Hongxia X ${ }^{1,2}$, Ying Ai ${ }^{1,2}$, Lingyun Su ${ }^{1,2}$, Li Zou $^{1,2}$, Linna Yang ${ }^{1,2}$, Dehong Yang ${ }^{1,2}$,
} Xuelan $\mathrm{Yan}^{1,2}, \mathrm{Na} \mathrm{Ma}^{1,2}$ and Wei Dong ${ }^{1,2}$

\begin{abstract}
Background: This study aims to investigate the influencing factors of pregnancy after laparoscopic oviduct anastomosis.

Methods: The data of 156 cases of laparoscopic oviduct anastomosis in our hospital were analyzed.

Results: The pregnancy rate decreased with age $(P<0.005)$. The pregnancy rate after six years of anastomosis was higher in those with ligation $(P<0.005)$. The postoperative pregnancy rate significantly increased in subjects with oviduct lengths of $>7 \mathrm{~cm}(P<0.01)$. The pregnancy rate of isthmus end-to-end anastomosis was higher $(P<0.005)$. The pregnancy rate after bilateral tubal recanalization was higher than that after unilateral tubal recanalization $(P<$ 0.005). The pregnancy rate after laparoscopic tubal ligation and laparoscopic anastomosis was higher than that of open tubal ligation and laparoscopic anastomosis $(P<0.005)$.

Conclusion: The pregnancy rate after laparoscopic oviduct anastomosis is higher in subjects below 35 years old, with a ligation duration of $<6$ years, and a length of oviduct of $>7 \mathrm{~cm}$, and those who underwent isthmus anastomosis and laparoscopic oviduct ligation and recanalization.
\end{abstract}

Keywords: Laparoscopic, Oviduct anastomosis, The pregnancy rate, Correlated factors

\section{Background}

With the full relaxing of China's second-child policy, more women who underwent tubal oviduct ligation want to recanalize the oviduct and get pregnant again. Thus, they face a choice of reoperation for oviduct recanalization. Oviduct anastomosis is an operation to reconstruct the structure and function of the oviduct after tubal ligation [1], and has been proved to be effective in younger women [2].

The clinical data of 156 women after oviduct anastomosis in our hospital were retrospectively analyzed. The factors that influenced pregnancy outcome, in terms of the age of the recipient, the duration of the sterilization period, the length of the oviduct after recanalization, and the method of oviduct anastomosis and previous

\footnotetext{
* Correspondence: fengyun078@126.com

${ }^{\dagger}$ Yun Feng and Han Zhao contributed equally to this work.

'Department of Obstetrics and Gynaecology, The First People's Hospital of Yunnan Province, No.157 of Jinbi road, Kunming 650032, China

${ }^{2}$ Medical School of Kunming University of Science and Technology, Kunming 650500, China
}

tubal ligation method, were investigated to provide the basis for evaluating the indications of oviduct anastomosis in clinical application.

\section{Methods \\ General information}

A total of 156 subjects, who underwent laparoscopic oviduct anastomosis in our department from January 2014 to October 2018, were enrolled as the study subjects. All subjects had a regular menstrual cycle. The age of these subjects ranged within 23-45 years old, with an average age of 35.04 years old. The duration of the sterilization period of these subjects ranged within 1-23 years, with an average duration of 8.77 years. Among these subjects, 92 subjects underwent open oviduct ligation, while 64 subjects underwent laparoscopic oviduct sterilization. All subjects were assessed for normal ovarian function and menstrual cycle before the operation. Subjects with uterine and appendage tumors were excluded. No surgical contraindication was

(c) The Author(s). 2019 Open Access This article is distributed under the terms of the Creative Commons Attribution 4.0 International License (http://creativecommons.org/licenses/by/4.0/), which permits unrestricted use, distribution, and 
found in these subjects. Furthermore, the semen examination results of their spouses were normal.

\section{Surgical methods}

Selection of time of operation: The operation was performed on the 3-10th day after the menstruation stopped. Surgical procedures: All subjects underwent laparoscopic oviduct anastomosis. Anesthesia procedure: Subjects were treated with general anesthesia and endotracheal intubation. Surgical methods: Subjects were placed in the lithotomy position, routine disinfection was performed, and the uterine cavity dual-lumen tube was placed for laparoscopic oviduct hydrotubation. The umbilicus was taken as the first puncture hole. The skin and part of the fascia was cut. A $10-\mathrm{mm}$ puncture device was punctured in. $\mathrm{CO}_{2}$ was used to form pneumoperitoneum. A laparoscope was placed to explore the abdominopelvic cavity. Two 5-mm incisions were made at the skin $5 \mathrm{~cm}$ to the lower left from the scar of original operation and theumbilicus. The $5-\mathrm{mm}$ puncture device was inserted into the abdominal cavity. The uterus, pelvic cavity and bilateral ovaries were observed. If lesions were present, these were treated, accordingly. The diluted methylene blue solution was injected through the double-lumen tube, and the oviduct sites where the methylene blue solution flowed through were observed. The serosal layer of the oviduct was incised according to different ligation sites. The bilateral broken ends of oviducts were freed. The scar tissues at the broken ends were cut off. Electrocautery was performed at the bleeding point. For subjects who underwent laparoscopic oviduct ligation with a titanium clip, the titanium clip was removed, and the closed ends of oviduct were cut off. Methylene blue solution was injected through the vaginal dual-lumen tube. It could be observed under a laparoscope that the methylene blue solution flowed smoothly from the proximal end. For this case, the distal oviduct lumen was positioned. Otherwise, an epidural catheter was sent to pass through the oviduct fimbria to the incision site, and three stitches of intermittent fullthickness suture of both broken ends of the oviduct were performed with 5/0 absorbable surgical suture. Then, the mesosalpinx was sutured.

\section{Criteria for determining the patency of the oviduct after anastomosis}

After performing the hydrotubation again, the methylene blue solution smoothly flowed out the oviduct fimbria. The length of the oviduct was not less than $6 \mathrm{~cm}$. If a small volume of methylene blue solution leaked out from the anastomosis site, reinforcement and suture were not required, since this would not affect the healing of the anastomosis and the pregnancy rate after the operation [2]. A small volume of normal saline (N.S) was injected into the pelvic cavity to keep the oviduct wet during the operation.

\section{Clinical curative effect evaluation}

At one month after the operation, the subjects were guided to naturally conceive and underwent followed ups. The pregnancy of subjects after the operation was recorded, and the factors that affected the pregnancy were analyzed.

The diagnostic criteria of pregnancy include: (1) dysmenorrhea; (2) blood test was positive for Human Chorionic Gonadotropin (HCG) and gynecological b-ultrasound indicated intrauterine pregnancy.

\section{Statistical methods}

The study data were statistically analyzed using statistical software SPSS 11.0. Intergroup comparison of count data was performed using $X^{2}$-test.

\section{Results}

\section{Surgical outcomes}

The bilateral oviducts became unobstructed in 127 subjects, while a single side of the oviduct became unobstructed in 29 subjects, and the patency rate was $100 \%$.

\section{Pregnancy of subjects after the operation}

A total of 106 subjects got pregnant after the recanalization, accounting for $67.94 \%$. Among these subjects, 99 subjects (99/127) got pregnant after bilateral oviduct recanalization, and the pregnancy rate was $77.95 \%$, while seven subjects (7/29) got pregnant after the unilateral oviduct recanalization, and the pregnancy rate was $24.14 \%(P<0.005)$. Furthermore, 54 subjects (54/92) who previously underwent open laparotomy oviduct ligation got pregnant after recanalization, and the pregnancy rate was $58.7 \%$, while 52 subjects (52/64) who previously underwent laparoscopic oviduct ligation got pregnant after recanalization, and the pregnancy rate was $81.2 \%(P<0.005)$. Moreover, three subjects (3/11) with an oviduct length of less than $7 \mathrm{~cm}$ got pregnant after anastomosis, and the pregnancy rate was $21.3 \%$, while 103 subjects (103/145) with an oviduct length of greater than $7 \mathrm{~cm}$ got pregnant after anastomosis, and the pregnancy rate was $71.0 \%(P<0.01)$ (Tables 1,2 and 3$)$.

Table $1 \mathrm{C}$ pregnancy rate after tubal anastomosis and the age of patient

\begin{tabular}{lll}
\hline Age (year) & Number of case & Rate of pregnancy(\%) \\
\hline$\leqq 35$ & 84 & $76.19 \%(64 / 84) \times 2: 8.32: P<0.005$ \\
$36-40$ & 56 & $67.85 \%(38 / 56) \times 2: 12.89: P<0.005$ \\
$>40$ & 16 & $25 \%(4 / 16)$ \\
Total & 156 & $67.94 \%(106 / 156)$ \\
\hline
\end{tabular}


Table 2 Relationship between sterilization duration and pregnancy rate after anastomosis

\begin{tabular}{lll}
\hline Duration (year) & Number of case & Rate of pregnancy(\%) \\
\hline$<6$ & 37 & $81.08 \%(30 / 37) \times 2: 12.59: P<0.005$ \\
$6-10$ & 73 & $79.45 \%(58 / 73) \times 2: 14.35: P<0.005$ \\
$11-14$ & 30 & $46.66 \%(14 / 30) \times 2: 1.66: P>0.1$ \\
$>15$ & 16 & $25 \%(4 / 16)$ \\
Total & 156 & $67.94 \%(106 / 156)$ \\
\hline
\end{tabular}

\section{Discussion}

In the present study, the data revealed that the repregnancy rate reached $76.19 \%$ in subjects with an age of $\leq 35$ years old after oviduct anastomosis, and this was higher than that in the other two groups $(P<0.005)$. This result suggests that the older the recipient, the lower the rate of re-pregnancy after anastomosis [3]. Therefore, the age at the time of recanalization is correlated to the postoperative pregnancy rate. Hence, it is particularly important to take into account both effective sterilization and the possibility of re-pregnancy [4]. However, age can affect fertility rates. So we should compare the data in our study with the data of the patients that are at the same age range of the patients in our study, but did not do laparoscopic oviduct anastomosis, so as to eliminate the effects of age.

The difference in length of the oviduct after oviduct anastomosis also directly affected the re-pregnancy rate of the subjects. The pregnancy rate was $71.0 \%$ in subjects with an oviduct length of $\geq 7 \mathrm{~cm}$. This was significantly higher than that in subjects with an oviduct length of $<7$ $\mathrm{cm}(P<0.01)$, and the difference was statistically significant. An oviduct length of $<5 \mathrm{~cm}$ can directly affect the fertilization and delivery of fertilized eggs, leading to a lower pregnancy rate. A short oviduct may cause these fertilized eggs to enter the uterine cavity too early, in which the development does not synchronize with the endometrial development, leading to spontaneous abortion or biochemical pregnancy [5].

In the present study, the re-pregnancy rate was $85.18 \%$ in subjects after isthmus-ampulla anastomosis of the oviduct, and this was significantly higher than that in the other groups, and significantly higher than that in subjects receiving ampulla-ampulla anastomosis of the oviduct $(39.58 \%, P<0.005)$. A study reported that the re-pregnancy

Table 3 Relationship between anastomotic site and pregnancy rate

\begin{tabular}{lll}
\hline Site & Number of case & Rate of pregnancy(\%) \\
\hline isthmus-isthmus & 81 & $85.18 \%(69 / 81)$ \\
isthmus-ampulla & 27 & $66.66 \%(18 / 27)$ \\
ampulla-ampulla & 48 & $39.58 \%(19 / 48) \times 2: 20.21: P<0.005$ \\
Total & 106 & $67.94 \%(106 / 156)$ \\
\hline
\end{tabular}

rate was higher in subjects after isthmus-isthmus anastomosis of the oviduct, when compared to that of subjects after anastomosis at other sites of the oviduct [6]. After the isthmus-ampulla anastomosis of the oviduct is performed, since the ampulla is rich in wrinkles, some oviduct mucosal wrinkles would often protrude outside of the anastomosis, which may affect the healing of the anastomosis, and subsequently affect the surgery [7].

The pregnancy rate after recanalization was higher in subjects who previously underwent laparoscopic oviduct ligation, when compared to subjects who previously underwent open oviduct ligation $(P<0.005)$. This was correlated to the characteristics of laparoscopic surgery, such as small incision, less abdominal interference, less tissue damage, and safety under direct vision [8]. A related study revealed that the incidence of adhesion after laparoscopic surgery was lower than that after open surgery [9]. Subjects who underwent oviduct ligation with a titanium clip had a longer retained segmental length and less damage to the oviduct. Oviduct lumen adhesion, stiffness, or traction, and the distortion or occlusion of the surrounding scar tissues [10] may cause the oviduct to lose its physiological function, such as the transport of sperm and eggs, and the fertilization of eggs.

At present, traditional open oviduct anastomosis, transabdominal microscopic oviduct anastomosis and laparoscopic oviduct anastomosis are the clinical methods of oviduct anastomosis. Microscopic oviduct anastomosis can anastomose the oviduct in a full-thickness, accurate, multi-needle and clear manner. Furthermore, the stent may not be placed, and fine needle and fine thread can be used to eliminate the stimulation and injury of foreign bodies to the oviduct mucosa, which practically avoids scars. Therefore, traditional concepts consider that only open surgery under a microscope can lead to a good prognosis [11-13]. However, with the improvement of laparoscopic microdevices and development of technology, an increasing number of literatures on laparoscopic oviduct anastomosis have been reported in recent years [14-16]. On a laparoscopically magnified TV screen, the cut ends of the oviduct are clearly displayed, making the oviduct anastomosis simple and easy under a laparoscope. Furthermore, the involution is good, and the characteristics of the laparoscopic operation, such as precision, meticulousness and accuracy, are fully applied. The oviduct mucosa is one of the most vigorously growing tissues among human tissues. Therefore, there is no need for excessive suture during surgery. In principle, as long as the lumens of the two ends are accurately aligned and the thickness is basically the same, the purpose can be achieved [17]. During the operation, attention should be given to the misalignment of the cut ends of the oviduct, which cannot be misplaced. If the diameter of the proximal end lumen is 
smaller than the distal end, the proximal oviduct can be cut in chamfer to preserve the oviduct tissue, as much as possible [18]. Furthermore, attention should be given to ensure that sufficient mesangial covers the surface of the wound of the oviduct after the anastomosis, in order to prevent adhesion formation [19].

Our strengths are that the sample size was not small, thus the result was convincing, and we found out some factors that can affect the effects of laparoscopic oviduct anastomosis, for example, ligation duration and length of oviduct. However, age per se can affect fertility rates, but we did not compare the data in our study with the data of the patients that are at the same age range of the patients in our study, but did not do laparoscopic oviduct anastomosis, which should be addressed in future studies. In addition, we did not compare the effects of laparoscopic oviduct anastomosis with in-vitro fertilization (IVF). Also, we did not evaluate the postoperative complications, which can be discussed in future studies. In addition, due to the long duration of pregnancy and limited trial time, the pregnancy outcomes of patients after fallopian tube anastomosis are still being followed up and no systematic data have been obtained, which can be mainly discussed in future studies. We have added it as a limitation of our study in the discussion section.

\section{Conclusion}

In summary, compared with open surgery, laparoscopic oviduct anastomosis has a clearer field of vision, more accurate involution and less interference, and the rate of re-adhesion is lower. However, there are many factors that affect the postoperative pregnancy of subjects. In addition, before surgery, the patient's age, duration of sterilization period and ovarian function should be fully evaluated, and the surgical actions need to be gentle, careful and accurate. Furthermore, the tissues separated and sutured during surgery should be as few as possible, and attention should be given to reduce injury to the mesangium, as much as possible, during surgery. When the serosa is sutured, care should be given to avoid twisting the oviduct. In addition, attention should be given to avoid damage to the oviduct cilia and anastomosis tissues, and reduce the scar formation of anastomosis, in order to facilitate the passage of fertilized eggs and improve the pregnancy rate.

\section{Abbreviations \\ HCG: Human Chorionic Gonadotropin; IVF: in-vitro fertilization; N.S: normal saline}

\section{Acknowledgements}

Not applicable.

\section{Authors' contributions}

Han Zhao and Wei Dong contributed equally to this work, drafted the initial manuscript, and reviewed and revised the manuscript.RL, AY,SLY,ZL, YLN,

YDH, DMF and WY designed the data collection instruments, collected data, carried out the initial analyses, and reviewed and revised the manuscript. DW coordinated and supervised data collection, and critically reviewed the manuscript for important intellectual content. All authors approved the final manuscript as submitted and agree to be accountable for all aspects of the work.

\section{Funding}

This study was supported by a grant from Projects: health science and technology plan projects of yunnan province (NO. 2017NS214, 2017NS215, 2017NS216, 2018NS0235, 2018NS0236).

Provincial innovation team project of human assisted reproductive technology research: 2017HC009. Martin expert workstation (2018IC106).

\section{Availability of data and materials}

The datasets used and/or analysed during the current study available from the corresponding author on reasonable request.

\section{Ethics approval and consent to participate}

This study was conducted in accordance with the declaration of Helsinki. This study was conducted with approval from the Ethics Committee of The First People's Hospital of Yunnan Provience. A written informed consent was obtained from all participants.

\section{Consent for publication}

Not applicable.

\section{Competing interests}

Not applicable.

Received: 8 April 2019 Accepted: 21 August 2019

Published online: 30 October 2019

\section{References}

1. Zhang J, Bai WP. Advances in the diagnosis and treatment of tubal infertility [J]. Maternity and heredity: electronic edition. 2014;4(1):56-60.

2. van Seeters JAH, Chua SJ, Mol BWJ, Koks CAM. Tubal anastomosis after previous sterilization: a systematic review [J]. Hum Reprod Update. 2017;23(3):358-70.

3. Karayalcin R, Ozcan S, Tokmak A, Gürlek B, Yenicesu O, Timur H. Pregnancy outcome of laparoscopic tubal reanastomosis: retrospective results from a single clinical Centre. [J]. J Int Med Res. 2017 Jun;45(3):1245-52.

4. He P, Wu N. Pregnancy effect and its influencing factors after tubal anastomosis in 117 cases [J]. Chinese eugenics and genetics. 2014;22(02):62-63+22.

5. Zhao Y Han B. Pregnancy rate and related factors after tubal anastomosis in 248 cases [J]. China Foreign Me. 2014;33(28):127-8.

6. Hou XH. Clinical analysis of salpingosalpingostomy in 198 cases [J]. Journal of Jinggangshan Medical College. 2009;16(5):35-6.

7. Liu JH, Wang XF, Ye X, Chen M, Fa YY. Effects of microsurgical tubal reversal after tubal sterilization [J]. J Shanghai Jiaotong Univ (Med Sci). 2006;(12):1369-72.

8. Chen SH. Comparison of curative effect between laparoscopic electrocoagulation ligation and open ligation [J]. Med Innov China. 2013; 10(28):28-30.

9. Ren XP, Xue JL. Comparison of postoperative adhesion between gynecological laparoscopic surgery and laparotomy [J]. Journal of Practical Medical Techniques. 2012;19(06):645-6.

10. Kirichenko AK, Khorzhevskii VA. Endometrial vasculature in women with hydrosalpinx [J]. Arkh Patol. 2014;76(3):59-64.

11. Wang JJ, Liu YH, Ying XY. Comparison of fertility rates between laparoscopic and open oviduct anastomosis [J]. Chinese Journal of Minimally Invasive Surgery, 2014, 14(8): 673-676. Chinese.

12. Cheng D, Yang J, Xie QZ, Li J. The clinical value of wholelayered suturing anastomosis of tube under laparoscope [J]. Chinese Journal of Laparoscopic Surgery: Electronic Edition, 2012, 5(6): 455-458. Chinese.

13. Lagana AS, Vitale SG, De Dominici R, Padula F, Rapisarda AM, Biondi A, Cianci S, Valenti G, Capriglione S, Frangez HB, Sturlese E. Fertility outcome after laparoscopic salpingostomy or salpingectomy for tubal ectopic pregnancy a 12-years retrospective cohort study [J]. Ann Ital Chir. 2016;87: $461-5$.

14. Zhou Z, Sheng L, Gui LH. Laparoscopic fallopian tube anastomosis with fallopian tube obstruction, 68 cases of clinical analysis [J]. Journal of Nanchang University (Medical Sciences), 2013, 53(8): 62-63. Chinese. 
15. Zhen WM, Zou L, Wu JY. Clinical effect evaluation of tubal anastomosis under laparoscopy [J]. Clinical Medicine, 2017, 37(6): 96-97. Chinese.

16. Dong F. Clinical operation of tubal anastomosis [J]. Guide of China Medicine, 2013, 11(16): 627-629. Chinese.

17. Xu J. Discussion on laparoscopic oviduct anastomosis and recanalization [ J]. Jiangxi Medical Journal. 2004;12:860.

18. Zhou HY, Zheng HF, Lv WC. Clinical application of laparoscopic oviduct anastomosis [J]. J Pract Obstet Gynecol. 2010;26(03):228-9.

19. Guan J, Shen H, Han HJ, Fang F. Laparoscopic tubal anastomosis: fertility outcome in 32 cases [J]. Chin J Clin Obstetrics GynEcol. 2007:252-5.

\section{Publisher's Note}

Springer Nature remains neutral with regard to jurisdictional claims in published maps and institutional affiliations.

Ready to submit your research? Choose BMC and benefit from:

- fast, convenient online submission

- thorough peer review by experienced researchers in your field

- rapid publication on acceptance

- support for research data, including large and complex data types

- gold Open Access which fosters wider collaboration and increased citations

- maximum visibility for your research: over $100 \mathrm{M}$ website views per year

At BMC, research is always in progress.

Learn more biomedcentral.com/submissions 